\title{
STAT3 Inhibitor DSP-0337
}

National Cancer Institute

\section{Source}

National Cancer Institute. STAT3 Inhibitor DSP-0337. NCI Thesaurus. Code C151994.

An orally administered prodrug of napabucasin, a small molecule cancer stemness inhibitor with potential antineoplastic activity. Upon administration, DSP-0337 is converted to its active form, napabucasin. Napabucasin targets and inhibits signal transducer and activator of transcription 3 (STAT3), thereby preventing ST AT -3mediated signaling. The STAT3 pathway is overly active in many cancer types and is implicated in cancer stem cell-mediated growth, recurrence and resistance to conventional chemotherapies. 